\title{
Principal component analyses in mungbean genotypes under summer season
}

\author{
Priya Tiwari* and Stuti Sharma \\ Department of Plant Breeding and Genetics, Jawaharlal Nehru Krishi Vishwa Vidyalaya, Jabalpur (M.P.) India \\ (E mail: tpriya725@gmail.com)
}

\begin{abstract}
Yield is a complex trait subjective to several components and environmental factors. Therefore, it becomes necessary to apply such technique which can identify and prioritize the key traits to lessen the number of traits for valuable selection and genetic gain. Principal component analysis is primarily a renowned data reduction technique which identifies the least number of components and explain maximum variability, it also rank genotypes on the basis of PC scores. PCA was calculated using Ingebriston and Lyon (1985) method. In present study, PCA performed for phenological and yield component traits presented that out of thirteen, only five principal components (PCs) exhibited more than 1.00 eigen value, and showed about 80.28 per cent of total variability among the traits. Scree plot explained the percentage of variance associated with each principal component obtained by illustrating a graph between eigen values and principal component numbers. PC1 showed 26.12 per cent variability with eigen value 3.40. Graph depicted that the maximum variation was observed in PC1 in contrast to other four PCs. The PC1 was further associated with the phenological and yield attributing traits viz., number of nodes per plant, number of pod cluster per plant, number of pod per plant. PC2 exhibited positive effect for harvest index. The PC3 was more related to yield related traits i.e., number of seeds per pod, number of seeds per plant and biological yield per plant, whereas PC4 was more loaded with phenological traits. PC5 was further related to yield and yield contributing traits i.e. number of primary branches per plant, seed yield per plant and 100 seed weight. A high value of PC score of a particular genotype in a particular PC denotes high value for those variables falling under that specific principal component. Pusa Vishal found in PC 2, in PC 3, PC 4 and PC 5, can be considered as an ideal breeding material for selection and for further deployment in defined breeding programme.
\end{abstract}

Key Words : Eigen values, Mungbean, Principal component analysis, Summer

View Point Article : Tiwari, Priya and Sharma, Stuti (2021). Principal component analyses in mungbean genotypes under summer season. Internat. J. agric. Sci., 17 (2) : 287-292, DOI:10.15740/HAS/IJAS/17.2/287-292. Copyright@2021: Hind Agri-Horticultural Society.

Article History : Received : 23.02.2021; Revised : 26.02.2021; Accepted : 15.03.2021

\footnotetext{
* Author for correspondence :
} 